\title{
BMJ Open Systematic review protocol examining the influence of surgeon personality on perioperative decision making in abdominal surgery
}

\author{
Carly Nichola Bisset (D) , ${ }^{1}$ Tracey McKee, ${ }^{2}$ Elliot Tilling, ${ }^{3}$ Mary Cawley, ${ }^{4}$ \\ Susan Moug ${ }^{1}$
}

To cite: Bisset CN, McKee T, Tilling E, et al. Systematic review protocol examining the influence of surgeon personality on perioperative decision making in abdominal surgery. BMJ Open 2020;10:e035361. doi:10.1136/ bmjopen-2019-035361

- Prepublication history and additional material for this paper are available online. To view these files, please visit the journal online (http://dx.doi org/10.1136/bmjopen-2019035361).

Received 30 October 2019 Revised 10 December 2019 Accepted 11 December 2019

Check for updates

(c) Author(s) (or their employer(s)) 2020. Re-use permitted under CC BY-NC. No commercial re-use. See rights and permissions. Published by BMJ.

${ }^{1}$ Department of General Surgery, Royal Alexandra Hospital,

Paisley, UK

${ }^{2}$ NHSGGC Library Network, Glasgow, UK

${ }^{3} \mathrm{NHS}$ Greater Glasgow and Clyde, Glasgow, UK

${ }^{4}$ West of Scotland Renal and Transplant Unit, Queen Elizabeth University Hospital, Glasgow, UK

Correspondence to Carly Nichola Bisset; cbisset@nhs.net

\section{ABSTRACT}

Introduction There is limited published literature exploring how the personality traits of surgeons may influence preoperative decision making, particularly in the context of visceral/abdominal surgery. Multiple validated personality scoring systems exist and have been used to describe surgeon personalities previously. The degree to which each trait is expressed by abdominal surgeons is neither currently known, nor the impact of these traits on postoperative outcomes. The protocol has been written in line with the Preferred Reporting Items for Systematic Review and Meta-Analysis Protocols checklist.

Methods and analysis The search strategy has been developed by a Health Scientist Librarian in collaboration with the review team. The search was conducted on 1st October 2019.

Database subject headings and text words relating to 'abdominal/general surgeons', 'personality', 'postoperative outcomes' and 'decision making' formed the basis of our literature search strategy; the MEDLINE, EMBASE, PsycInfo and Cochrane databases will be searched. Three reviewers will independently screen and appraise articles, with a fourth reviewer utilised if disagreements arise.

A systematic narrative synthesis will be performed, with information presented in text and table format. These will summarise the findings and characteristics of any included studies. Using guidance from the Centre for Reviews and Dissemination, the reviewers will describe the potential relationship and findings between studies using the narrative synthesis. Studies will only be reported if they are felt to have low or mid-levels of bias. Studies felt to display high levels of bias will be excluded.

Ethics and dissemination This study does not require ethical approval. The formal systematic review will be submitted for peer reviewed publication and presented at relevant conferences. The methods may inform future reviews in other surgical specialties regarding surgeon personality.

PROSPEROregistration number CRD42019151375.

\section{INTRODUCTION}

There is limited published literature exploring how the personality traits of surgeons may influence preoperative decision making. ${ }^{1}$ Abdominal surgery especially has a paucity of
Strengths and limitations of this study

- This systematic review develops consensus on the influence of abdominal surgeon personality on perioperative decision making.

- Design and delivery of systematic review by experienced review team and strict adherence in reporting as per Preferred Reporting Items for Systematic Review and Meta-Analysis Protocols guidelines.

- Articles will only be included if written in the English language, therefore excluding studies in other languages which may introduce bias.

such information. Several validated personality scoring systems exist, including the fivefactor model proposed by Gosling et al which includes the following domains: agreeableness, conscientiousness, emotional stability, extraversion and openness to experience. ${ }^{2}$ The degree to which each trait is expressed by abdominal surgeons is neither currently known, nor the impact of these traits on postoperative outcomes.

There is growing global interest in how the personality of the surgeon may affect perioperative decision making. Surgeon personality is hypothesised to affect patient morbidity and mortality through multiple mechanisms. ${ }^{3}$ We hypothesise that low scores in openness and emotional stability may influence surgical decision making, which may detrimentally affect morbidity and mortality rates for both medical and surgical complications. Personality traits which predispose rudeness or hostility may detrimentally affect team working and create a negative working environment, which may lead to concerns going unreported, and potentially poorer patient outcomes. ${ }^{5}$ Our systematic review was first registered on PROSPERO on 28 September 2019 (CRD42019151375). 


\section{OBJECTIVES}

The primary objective of this review is to summarise the limited existing literature to answer the question: Does surgeon personality influence perioperative decision making in abdominal surgery? Our secondary objective is to determine if surgeon personality affects post-operative patient outcomes following abdominal surgery.

\section{METHODS AND ANALYSIS}

\section{Eligibility Criteria-Study Characteristics}

This systematic review will include randomised and nonrandomised controlled trials and cohort studies. Casecontrol series, cross-sectional studies and case reports will be excluded as these are difficult to draw conclusions from in single population psychological studies. Animal studies will be excluded. The search was conducted on October 2019.

\section{Types of participants}

Participants must be abdominal (general) surgeons, therefore studies in specialities including gynaecology, vascular and urology will be excluded. Paediatric surgery will be excluded. Veterinary medicine studies will also be excluded. A participating surgeon for the study must be an 'independent practitioner' - that is, making independent perioperative decisions regarding surgery. This may therefore include some trainees depending on their competency.

\section{Setting and time frame}

Studies which do not include outcomes as part of their findings will not be excluded as these may contribute towards the primary objectives. Those studies which include postoperative outcomes will be included for analysis of the secondary objectives. For all decision-making postoperative outcomes, studies should have a minimum follow-up time of 30 days follow-up. Decisions may include any which alter the course of the initially intended operation; for example change of operation (eg, anterior resection to Hartmann's for rectosigmoid cancer), decision to form a stoma, or conversion to open from laparoscopic surgery.

\section{Report characteristics}

There is no limitation on date of publication. Articles will be included where abstracts are published or in press. The literature search will be limited to studies published in the English language, human subjects and surgeon personality only (not patient personality).

\section{Information sources}

Database subject headings and text words relating to 'abdominal/general surgeons', 'personality', 'postoperative outcomes' and 'decision making' formed the basis of our literature search strategy. The following databases were used: MEDLINE (OVID interface, 1948 onwards), EMBASE and Embase Classic (OVID interface, 1947 onwards), PsycInfo (OVID interface 1806 onwards) and
Cochrane Library (Wiley interface, current issue). The databases were searched by a Health Scientist Librarian, with prior systematic review experience.

To ensure literature saturation, the reference lists of included studies will be reviewed in order to include any further relevant studies. Our complete reference list of included articles will be submitted around the systematic review team, to ensure that no studies are missing which the review team may know of.

\section{Search strategy}

The search strategy was developed by the Health Scientist Librarian in collaboration with the review team, a second librarian acting as a peer reviewer (online supplementary appendix 1).

\section{STUDY RECORDS \\ Data management}

Once the initial search has been carried out, abstracts will be added to the database Refworks, which all reviewers will have communal access to. A separate Excel spreadsheet will be used by the authors to record inclusion (yes/no) in the systematic review. If the abstract is to be excluded, a fifth column labelled 'rationale' will allow the reviewer to explain why exclusion was necessary according to the study protocol. If there are disagreements as to whether an abstract should be included, the three reviewers will discuss (CNB, ET and SM). If consensus is not achieved, then a fourth reviewer will be asked to make the final verdict (MC). The spreadsheet will be checked to ensure duplicate studies are not included, scrutinising each field.

Questions regarding study eligibility may be answered by directly contacting the study authors if further information is required. Reviewers will not be blinded to the authorship or journal.

\section{SELECTION PROCESS}

Literature search results will be uploaded to RefWorks, an internet-based reference management software programme, which acts as a database for the team to manage articles throughout the review. The inclusion and exclusion criteria will be applied to these articles in order to test screening questions as a pilot study. An Excel spreadsheet will be kept for reviewers to record decisions made about the inclusion or exclusion of articles, with an explanation regarding the reviewers reasoning.

\section{Data items}

Authors CB, ET and SM will independently screen the titles and abstracts of those articles yielded by the search using our inclusion criteria. The three reviewing authors will then appraise the full text of each article to determine if the inclusion criteria have in fact been met. Where there is uncertainty regarding eligibility, a fourth author (MC) will be invited to discuss each case to reach a conclusion, with the reasons for exclusion being recorded. 


\section{Data extraction}

Data extraction from the full text articles (as detailed above) will be recorded on a Microsoft Excel spreadsheet, containing fields such as:

- Study details (title, year of publication and authorship)

- Study design (type of study)

- Participant demographics (eg, colorectal surgeon and experience of surgeon)

- Personality index tool adopted (eg, Gosling ten item personality index)

- Secondary outcomes (eg, 30-day mortality in relation to personality test score)

- Results (raw data on surgeon personality scores and secondary outcome measurements)

\section{Outcomes and prioritization}

The primary outcome will be determining if surgeon personality testing has been undertaken using a validated test for example, Gosling index with absolute personality trait scores reported.

Secondary outcomes include:

- Determining postoperative 30-day patient outcomes (eg, morbidity and mortality) in relation to surgeon personality test score

- Determining changes in perioperative decision making (eg, stoma formation and conversion to open surgery from laparoscopic) in relation to surgeon personality test score

\section{Risk of bias in individual studies}

To combat the risk of bias present in each study, the Newcastle-Ottawa scale or Cochrane risk of bias will be applied. Each domain included in the tool will be accounted for, with a description regarding how the authors judged the risk of bias per study. This will include 'high-risk' or 'low-risk' judgements on level of bias within each study, using a traffic light system to act as a visual tool regarding their reliability. Insufficient information regarding an article will render the bias risk 'uncertain', prompting contact with the original study authors to ascertain further information. These judgements will be made independently by the three reviewing authors (CNB, ET and SM). Any disagreements will be discussed with a fourth author (MC), and if unresolved the remaining collaborating authors will also aid discussion to reach a conclusion. We anticipate that bias in this systematic review will most likely relate to self-selection bias of volunteering surgeons participating in personality testing studies.

\section{Data synthesis}

A systematic narrative synthesis will be provided with information presented via text and tables to summarise the findings of included studies, as well as details regarding the surgeons contributing to the data. The narrative synthesis will explore the relationship and findings between studies, in accordance with guidelines from the Centre for Reviews and Dissemination. ${ }^{7}$

\section{Meta-Biases}

Meta-analysis would be impossible in this setting due to the anticipated variety in participating surgeon groups and personality index tools used. Studies will only be reported if they are felt to have low or mid-levels of bias. Studies felt to display high levels of bias will be excluded.

\section{Confidence in cumulative evidence}

All included studies will be assessed in order to determine the unit of postoperative outcome measurement, to ensure this is consistent for analysis as a group. Where there is missing data, the reviewers will attempt to contact the original authors to clarify. All studies which have participating surgeon groups will be included-there will not be a participant attrition cut-off point. Clinical heterogeneity will be tested where possible to include participant demographics such as age and gender. It is predicted that even with the defined key words to include abdominal surgeons, clinical heterogeneity will persist and thus may not be appropriate to perform quantitative synthesis.

We predict that small-study effects may be seen in the articles which will reach our inclusion criteria, increasing the likelihood of sampling bias. If study protocols are available, the reviewing authors will compare the protocol with the reported outcomes to determine if publication bias is present. As such, the Outcome Reporting Bias in Trials (ORBIT) classification system to assess reporting bias.

\section{Ethics and dissemination}

The results from the formal systematic review will be submitted for publication in a peer reviewed journal and will be submitted for presentation at relevant surgical conferences. The methods may be used to inform future reviews in other surgical specialties regarding surgeon personality.

\section{Patient and public involvement}

Patients or the public were not involved in the design, conduct, reporting or dissemination plans of this systematic review protocol.

\section{DISCUSSION}

The role of surgeon personality and its potential influence on perioperative decision making in abdominal surgery is still unclear. This has been investigated somewhat previously between the different abdominal surgical specialties (eg, hepato-biliary, bariatric and colorectal), ${ }^{8-10}$ however has not been synthesised in a meaningful way. Understanding the role of surgeon personality on decision making and outcomes is an important research question which remains unanswered thus far and may vary between surgical specialties, hence this review's focus on abdominal surgery. Currently, wide heterogeneity exists within operative decision-making literature due to various surgeon populations, surgeon demographics and study 
settings. Therefore, this systematic review may clarify the exact role of specific personality traits on perioperative decision making in abdominal surgery, and may better inform future reviewers of appropriate, validated tools to report personality traits. Identification of such validated personality tools may be adopted by future studies examining decision making in surgery. This may lead to the future study of heuristics in surgery, which explores the cognitive biases made by surgeons in their decisionmaking processes.

Twitter Carly Nichola Bisset @CBizzle1 and Susan Moug @susanmoug

Contributors CNB and SM conceived the idea of the study. CNB, TM and SM were responsible for the design of the systematic review. TM conducted the literature search. CNB, SM and ET were involved in the screening process with MC acting as an independent reviewer. CB prepared the initial protocol draft with input from TM, $\mathrm{MC}$ and SM. All authors contributed to the drafting and subsequent final revision of this protocol manuscript.

Funding The authors have not declared a specific grant for this research from any funding agency in the public, commercial or not-for-profit sectors.

Competing interests None declared.

Patient consent for publication Not required.

Provenance and peer review Not commissioned; externally peer reviewed.

Open access This is an open access article distributed in accordance with the Creative Commons Attribution Non Commercial (CC BY-NC 4.0) license, which permits others to distribute, remix, adapt, build upon this work non-commercially, and license their derivative works on different terms, provided the original work is properly cited, appropriate credit is given, any changes made indicated, and the use is non-commercial. See: http://creativecommons.org/licenses/by-nc/4.0/.

ORCID iD

Carly Nichola Bisset http://orcid.org/0000-0003-3331-4420

\section{REFERENCES}

1 Whitaker M. The surgical personality: does it exist? Ann R Coll Surg Engl 2018;100:72-7.

2 Gosling SD, Rentfrow PJ, Swann WB. A very brief measure of the Big-Five personality domains. J Res Pers 2003;37:504-28.

3 Preece RA, Cope AC. Are surgeons born or made? A comparison of personality traits and learning styles between surgical trainees and medical students. J Surg Educ 2016;73:768-73.

4 McGreevy J, Wiebe D. A preliminary measurement of the surgical personality. The American Journal of Surgery 2002;184:121-5.

5 Riskin A, Erez A, Foulk TA, et al. Rudeness and medical team performance. Pediatrics 2017;139:e20162305.

6 Cooper WO, Spain DA, Guillamondegui O, et al. Association of coworker reports about Unprofessional behavior by surgeons with surgical complications in their patients. JAMA Surg 2019;154:828.

7 Centre for Reviews and Dissemination, University of York. Guidance: systematic reviews ISBN 1900640473, 2009.

8 Dekker SWA, Hugh TB. Laparoscopic bile duct injury: understanding the psychology and heuristics of the error. ANZ J Surg 2008;78:1109-14.

9 Shubeck SP, Kanters AE, Dimick JB. Surgeon leadership style and risk-adjusted patient outcomes. Surg Endosc 2019;33:471-4.

10 Moug SJ, Henderson N, Tiernan J, et al. The colorectal surgeon's personality may influence the rectal anastomotic decision. Colorectal Disease 2018;20:970-80. 